\title{
A new approach to corpus callosum anomalies in idiopathic scoliosis using diffusion tensor magnetic resonance imaging
}

\author{
Olivier Joly • Dominique Rousié • Patrice Jissendi • \\ Maxime Rousié • Edit Frankó
}

Received: 10 December 2013/Revised: 21 June 2014/ Accepted: 21 June 2014/Published online: 2 July 2014

(C) The Author(s) 2014. This article is published with open access at Springerlink.com

\begin{abstract}
Purpose Idiopathic scoliosis (IS) is a frequent 3D structural deformity of the spine with a multi-factorial aetiology which remains largely unclear. In the last decade, human magnetic resonance imaging (MRI) morphometry studies (e.g. cortical thickness, 2D shape of the corpus callosum) have aimed to investigate the potential contribution of the central nervous system in the etiopathogenesis of IS. Recent developments in diffusion tensor imaging (DTI) allow us to extend the previous work to the study of white matter microstructure. Here, we hypothesized that part of the corpus callosum could show a difference in white matter microstructure in IS patients as compared to healthy controls.
\end{abstract}

O. Joly and D. Rousié contributed equally to this work.

Electronic supplementary material The online version of this article (doi:10.1007/s00586-014-3435-3) contains supplementary material, which is available to authorized users.

\footnotetext{
O. Joly $(\bowtie)$

Institute of Neuroscience, Newcastle University,

Newcastle NE2 4HH, UK

e-mail: olivier.j.joly@gmail.com

D. Rousié $(\square)$

Service de chirurgie maxillo-faciale, Centre Hospitalier

Regional Universitaire de Lille, Lille, France

D. Rousié

Chirurgie Orthopedique, Clinique Lille Sud, 96 rue Gustave

Delory, 59810 Lesquin, France

P. Jissendi

Service de Neuroradiologie, IRM 3T Recherche, Plateforme Imagerie du Vivant, IMPRT-IFR 114, Centre Hospitalier

Regional Universitaire de Lille, Lille, France
}

Methods We acquired DTI in 10 girls with IS and in 49 gender-matched controls to quantify the fractional anisotropy (FA) along the corpus callosum.

Results Despite a very similar pattern of FA along the corpus callosum (maxima in the splenium and the genu and minimum in the isthmus), we found a significantly lower FA in the body in patients with IS as compared to control subjects. This region is known to connect the motor and premotor cortices of the two hemispheres.

Conclusion This first diffusion magnetic resonance imaging brain study in IS patients, suggests that differences in white matter development, such as synchronization of axonal myelination and pruning could be involved in the etiopathogenesis of IS.

Keywords Idiopathic scoliosis - Diffusion tensor imaging · Corpus callosum · Fractional anisotropy

M. Rousié

Universite libre de Bruxelles, Brussels, Belgium

E. Frankó

Department of Neurodegenerative Disease,

Institute of Neurology, University College London,

London WC1N 3BG, UK

E. Frankó

National Prion Clinic, National Hospital for Neurology and Neurosurgery, University College London Hospitals, London WC1N 3BG, UK 


\section{Introduction}

Idiopathic scoliosis (IS) is a developmental disorder characterized by a 3D structural deformity of the spine that arises in $1-3 \%$ of children [1]. IS affects more often girls than boys and it typically progresses during puberty. As its name suggests, the aetiology of IS is largely unknown although genetic factors [2] are known to play a role (see [3] for a review). Beside scoliosis, neurological dysfunctions have often been observed in IS patients, including vestibular [4, 5], postural $[5,6]$ and locomotor [7] deficits. These symptoms suggest that a defect of processing by the central nervous system may contribute in the aetiopathogenesis of IS. Despite the difficulty to identify a causal relationship [8] between these neurological dysfunctions and IS, further characterization of functional and morphological changes in the brain would improve our understanding of the disease.

To better understand the putative role of the different parts of the central nervous system in IS, brain imaging has been used to seek both morphological $[9,10]$ and functional [11] differences between patients and healthy controls. Three previous studies reported morphological differences, however, their results still remain difficult to combine or unify. In particular, while Liu and colleagues [12] reported a larger corpus callosum (CC) in right thoracic IS than in controls, Lee et al. [13] found no significant difference between groups, whereas Wang and colleagues [9] reported a morphometric abnormality in the splenium of the CC in the left thoracic IS. These previous studies suggest that the corpus callosum, the major fibre tract that connects the two cerebral hemispheres, might show abnormal morphology in IS but a direct investigation of its white matter microstructure has not yet been performed.

To investigate in vivo the microstructure of the white matter within the corpus callosum in humans, the method of choice is diffusion magnetic resonance imaging, a technique sensitive to the microscopic motion of water molecules [14] which is also becoming a common clinical tool for white matter disorders (e.g. stroke, callosal dysgenesis). Diffusion tensor imaging (DTI) can be used to determine the orientation of cerebral white matter fibres [15] and to derive the fractional anisotropy (FA) within each voxel of the image [16]. FA is calculated from the eigenvalues of the diffusion tensor. It is a scalar value between zero and one that describes the degree of directionality of water diffusion through tissue.

In the present study, we used DTI in 10 girls with IS and 49 controls to compare quantitatively the white matter microstructure of the corpus callosum within both populations. We hypothesized that the $\mathrm{CC}$ which connects the two hemispheres shows changes in the white matter microstructure which might be associated with the morphometric abnormality previously reported in the splenium [9].

\section{Materials and methods}

\section{Subjects}

Ten girls with IS were recruited (10-24 years of age, median age 14, right-handed) from the CHRU Hospital of Lille. The scoliosis and its progression were assessed with a repeated measurement at 6 months of the degree of angulation measured with EOS 3D reconstructions [17]. The deformity was considered progressive if it increased by at least three degrees at the thoracic level. Neuro-developmental abnormalities were further assessed from T2-weighted MRI in all subjects. Patients with congenital or juvenile idiopathic scoliosis, or secondary scoliosis (e.g. following skeletal, muscular or nervous system pathology) were excluded from the study. Inclusion criteria for the patient group were Cobb angle $\geq 15^{\circ}$ (see Table 1). All the patients in this study belong to the two most common types: right thoracic and right thoracic-left lumbar. The regional research ethics committees (CPP, 2009-A01050-57) approved the study and informed written consent was obtained from all patients and their parents. Data from 49 (10-18 years of age, median age 14) right-handed healthy girls as control subjects were obtained from the NIH Pediatric MRI Data Repository created by the NIH MRI Study of Normal Brain Development.

\section{Magnetic resonance imaging}

In patients, magnetic resonance images were acquired at 3 Tesla (Achieva, Philips Medical Systems) using parallel imaging SENSE-Head-8 channels coil at the University Hospital of Lille, without sedation or general anaesthesia.

Table 1 Characteristics of subjects

\begin{tabular}{llllll}
\hline $\begin{array}{l}\text { Subject } \\
\text { no }\end{array}$ & Hand. & $\begin{array}{l}\text { Age } \\
\text { (years })\end{array}$ & $\begin{array}{l}\text { Cobb angle } \\
\left({ }^{\circ}\right)\end{array}$ & Type & Progression \\
\hline 01 & $\mathrm{R}$ & 17 & 56 & $\mathrm{TL}$ & +++ \\
02 & $\mathrm{R}$ & 14 & 72 & $\mathrm{TL}$ & +++ \\
03 & $\mathrm{R}$ & 16 & 20 & $\mathrm{~T}$ & + \\
04 & $\mathrm{R}$ & 13 & 30 & $\mathrm{TL}$ & + \\
05 & $\mathrm{R}$ & 12 & 28 & $\mathrm{~T}$ & + \\
06 & $\mathrm{R}$ & 24 & 26 & $\mathrm{TL}$ & + \\
07 & $\mathrm{R}$ & 10 & 75 & $\mathrm{TL}$ & +++ \\
08 & $\mathrm{R}$ & 21 & 32 & $\mathrm{TL}$ & + \\
09 & $\mathrm{R}$ & 23 & 63 & $\mathrm{TL}$ & + \\
10 & $\mathrm{R}$ & 10 & 26 & $\mathrm{~T}$ & + \\
\hline
\end{tabular}

This table gives for the IS subjects the following information: handedness (Hand.), age, Cobb angle, type (T: right thoracic, TL: right thoracic-left lumbar,) and progression of the scoliosis 
The sequences included a T1-weighted 3D acquisition, with echo time $(\mathrm{TE})=3.301 \mathrm{~ms}$, flip angle $=9^{\circ}$, repetition time $(\mathrm{TR})=7.199 \mathrm{~ms}$, slice thickness $=1 \mathrm{~mm}$, matrix $=256 \times 256$ and a DTI spin-echo echoplanar image (SE-EPI) 78 axial slices, slice thickness $=2 \mathrm{~mm}$, $\mathrm{TR}=13,000 \mathrm{~ms}, \quad$ flip $\quad$ angle $=90^{\circ}, \quad \mathrm{TE}=55 \mathrm{~ms}$, matrix $=128 \times 128$ and along 32 isotropically distributed directions with $b$ values of $1,000 \mathrm{~s} / \mathrm{mm}^{2}$. The DTI acquisition was performed with isotropic resolution of $2 \mathrm{~mm}$. The MR acquisitions in patients lasted for about $30 \mathrm{~min}$ (10 and $20 \mathrm{~min}$ for the T1 and the diffusion MR sequence, respectively).

\section{NDAR Pediatric Dataset}

Data from controls were downloaded from the NDAR Pediatric Dataset website. The data included a T1-weighted MRI and tensor derived images including the FA maps which were acquired at 1.5 Tesla with 6 directions. The diffusion derived images had isotropic voxels of $2 \mathrm{~mm}$. To validate the compatibility of the two datasets, we also acquired in one patient a DTI spin-echo echoplanar image at 1.5T (GE Medical Systems Signa) with isotropic resolution of $2.5 \mathrm{~mm}$.

\section{Data analyses}

MR images from patients were first converted from DICOM to NIFTI format using the dcm2nii program that is distributed with MRIcron software. Then, diffusion images were processed to remove image distortion that arises from the effect of eddy currents on the EPI readout using eddycorrect function of the FDT software (FMRIB's Diffusion Toolbox available in FSL software http://fsl.fmrib.ox. ac.uk). The FDT was also used to generate the diffusion tensor for each voxel, from which FA was derived. FA image was then thresholded $(\mathrm{FA}>0.25)$ to avoid voxels that are not part of the white matter tract and minimize inclusion of voxels with a high degree of partial volume effect [18]. SPM8 (Statistical Parametric Mapping software, www.fil.ion.ucl.ac.uk) was used to compare the two groups at the voxel-based level. Prior to the statistical analysis, all the MR data were co-registered (affine registration with fast cross-correlation) to the standard MNIspace using ANTS (Advanced Normalization ToolS) software (www.picsl.upenn.edu/ANTS) and were smoothed with a Gaussian kernel $5 \mathrm{~mm}$ FWHM (full width at half maximum). In SPM, we tested for FAs being lower in IS group than in controls ( $p<0.05$, family-wise error correction). Statistical maps were overlaid onto the singleT1 template available in SPM. Although "radial" and "axial" diffusivities can be easily derived from the eigenvalues of the diffusion tensor, their interpretations are a matter of debate; therefore, we decided to focus on the fractional anisotropy only. Indeed, in a previous report [19], the authors discourage from interpreting changes of the "axial" and "radial" diffusivities on the basis of the underlying tissue structure.

\section{ROI analysis and DTI template}

We used the ICBM-DTI-81 (International Consortium of Brain Mapping) atlas as a template (http://www.loni.ucla. edu/Atlases/). ICBM-DTI-81 is a white matter atlas based on probabilistic tensor maps obtained from 81 normal right-handed adults ( $18 \leq$ age $\leq 59,39$ females). Three main regions (splenium, body and genu) of the corpus callosum were selected amongst the 50 white matter tract labels of the atlas. The body of the corpus callosum was further sub-divided (antero-posterior dimension) in three regions according to the modified Witelson scheme defined earlier [20] (Fig. 1). From posterior, these regions are the isthmus (IV) with posterior parietal, superior temporal projections, the posterior midbody (III) with primary motor cortex projections and the anterior midbody (II) with projections to the premotor and supplementary motor cortical areas [20]. This subdivision of the body is illustrated in Fig. 1 and was further limited to $x=[-12 ; 12] \mathrm{mm}$ in lateral dimension. The FA maps in patients were compared with those in controls using Mann-Whitney test. This nonparametric test was justified because of the relatively small sample size in the patient group and the non-Gaussian distribution of the FA values. The threshold for statistical significance was set at $p=0.05$ and significance level was corrected for multiple comparison (number of ROIs) using Bonferroni correction.

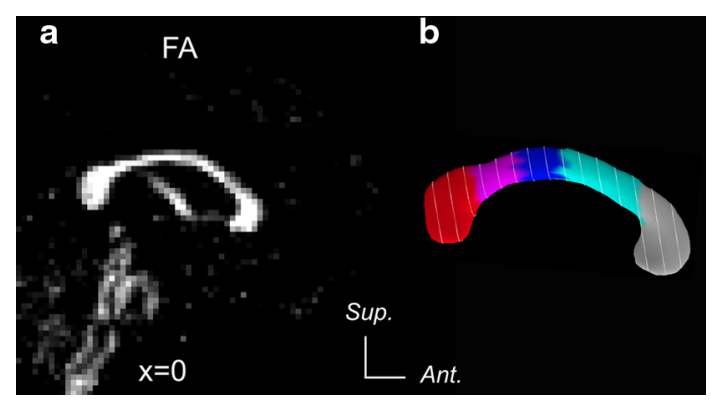

Fig. 1 a Midsagittal view of an example FA map from a control subject. b Lateral view of the (3D) corpus callosum and its subdivisions according to [20]. From posterior to anterior: splenium region (red); body regions including: isthmus region (purple), posterior midbody (blue) and anterior midbody (turquoise); and genu region (grey). White lines represent coronal sections at $5 \mathrm{~mm}$ steps. 3D file is also available online at http://brainsenses.x10host.com/ ICBM_CC.pdf 


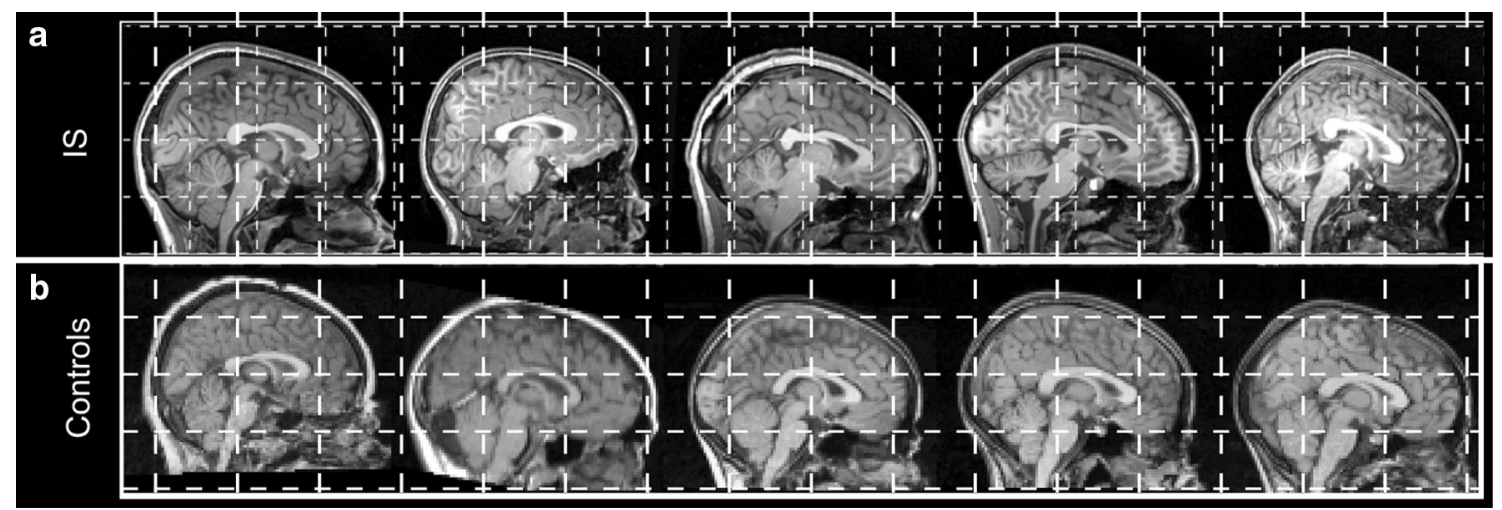

Fig. 2 Midsagittal slices of T1-weighted MRIs in 5 patients (a) and in 5 controls (b)

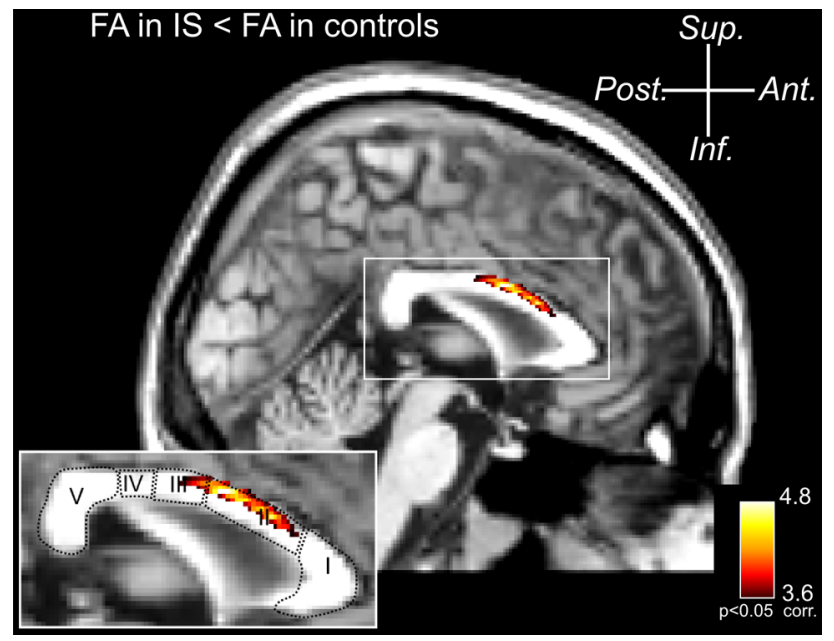

Fig. 3 SPM results (voxel-based analysis) for lower FA values in IS patients as compared to controls. Overlay of statistical maps ( $p<0.05$ FWE-corr.) onto the anatomical template at $x=0$ (midsagittal plane) showing significant voxels in region II of the CC

\section{Results}

In this study, we analysed the fractional anisotropy in patients with IS and healthy controls. Prior to the analyses of FA in the two populations, it is useful to visualize their T1 weighted anatomical MRI. In Fig. 2, we display the midsagittal views of the T1-weighted MRIs from 5 patients (Fig. 2a) and from 5 randomly selected controls (Fig. 2b). To appreciate the differences in size of the head and the corpus callosum, the T1-MRIs were co-registered to MNIspace with rigid only transformation. The head sizes differed across subjects as our population varies in age. The shapes of the $\mathrm{CC}$ also showed inter-individual variability.

The fractional anisotropy map for the midsagittal view of a control subject's corpus callosum (greyscale ranging from black for FA $<0.2$ to white for FA $=0.8$ ) is shown in Fig. 1a. Subdivisions of the corpus callosum as defined by Hofer and Frahm [20] is illustrated in Fig. 1b. The most pronounced anisotropy is found in the anterior and posterior regions of the $\mathrm{CC}$ with the highest $\mathrm{FA}$ values close to 0.8 in the most posterior area. The lowest FA values were between 0.25 and 0.5 and were located in the middle region.

Using a voxel-based two-sample $t$ test analysis implemented in SPM, we looked for significantly lower FAs in IS patients as compared to controls (Fig. 3). The map revealed significant voxels in the anterior part of the midbody of the corpus callosum which corresponds mainly to the region II from the modified Witelson scheme [20].

To complement the voxel-based analysis, ROI-based analysis was also performed (see "Materials and methods"). The distribution of FA values is shown in Fig. 4 for the five regions of interest illustrated in Fig. 1 along the antero-posterior dimension of the corpus callosum. All the individuals from both populations are represented (red crosses for controls, blue filled circles for IS patients). We also plotted the average FA values derived from the ICBMDTI-81 adult template. We found that both for the IS patients and the controls, the overall distribution of FA along the CC followed the profile of the DTI adult template. However, despite the similar pattern of FA in both the patient and control groups, a significantly lower FA in patients was found in the two parts of the midbody (section III; $p=0.0003$ and II; $p=0.0002$ ). This pattern corroborates the results observed in the voxel-based analysis (Fig. 3) in which the significant voxels were found in the section II of the partition of the corpus callosum. This region II also shows the maximum statistics for difference between the two populations amongst the region of interests. Note that according to the region of interests analysis, the two subdivisions of the midbody show significantly lower FA in IS than controls. Although, our voxel-based analysis might have failed detecting the effect in the posterior body, taken together both analyses suggest a lower FA in IS than controls in the body of the corpus callosum and especially in its most anterior part. 
Fig. 4 Distribution of Fractional Anisotropy along the corpus callosum in the 10 IS patients (blue circle for individual subjects) and 49 controls (red crosses for individual subjects). Box plots show the lower to upper quartile values and the median. The black dotted line represents the FA values of the ICBM-DTI-81 adult template

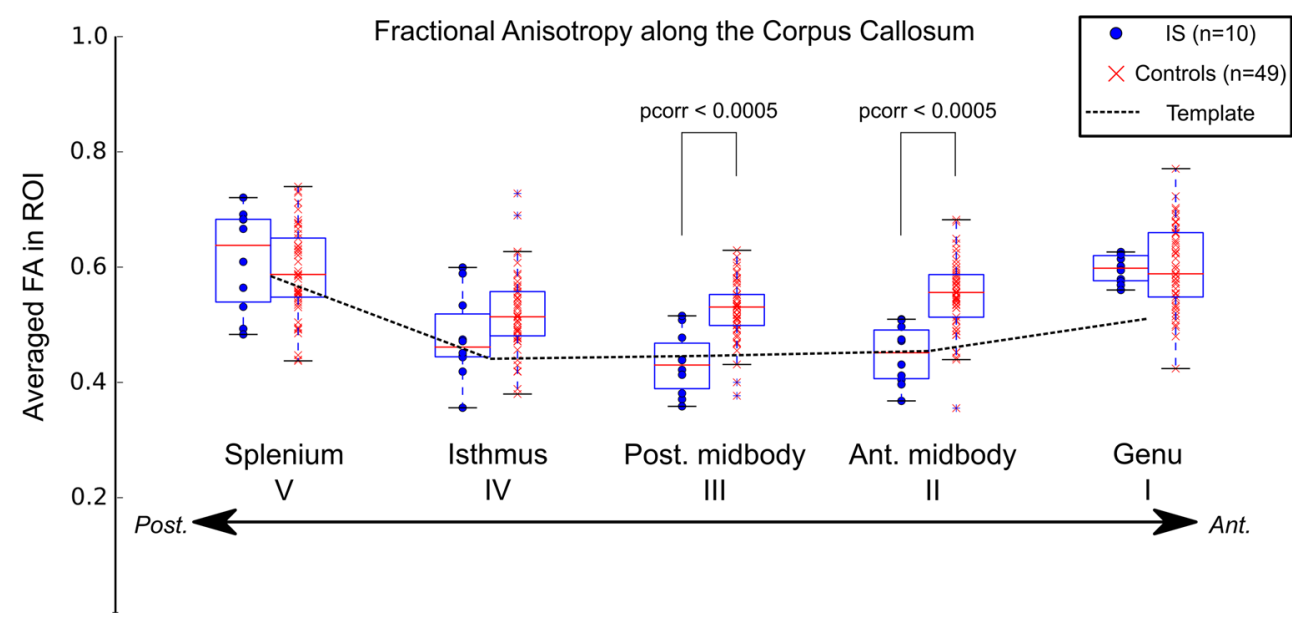

\section{Discussion}

In the present study, we measured DTI-derived quantity along the corpus callosum to evaluate the white matter microstructure in 10 young females with IS as compared to 49 sex-matched controls. We found that the overall distribution of FA along the CC was very similar in both populations. Moreover, it followed the profile derived from the adult ICBM-DTI-81 atlas. However, we found that the body had significantly lower FA in patients with IS than in controls (Figs. 3, 4). In particular, the anterior part of the body (region II) showing a significant difference in both the voxel and ROI-based analyses.

Fractional anisotropy describes how homogeneous is the diffusion of water molecules in one voxel. However, the water diffusion, and ,therefore, the value of FA, are sensitive to different tissue properties, such as axon diameter, packing density, membrane permeability, myelination or number of axons in a voxel. Therefore, one must be cautious when interpreting a difference in FA, as many white matter microstructure changes may have similar effect on these measurements [21, 22]. Brain development is characterized by both progressive myelination and regressive pruning processes. These two factors can modulate substantially the FA values. Myelination increases the outer diameter of the axon and decreases its permeability, therefore, increasing the FA. In contrast, elimination of axons during development (pruning) decreases the packing density and the number of axons in a voxel, therefore, decreasing FA. These two processes are particularly active during childhood and adolescence. They depend on genetic and environmental factors, such as the level of electrical activity of an axon [23, 24] and on experience [25]. Previous studies showed significant pruning in the corpus callosum during development of rhesus monkeys resulting in 3.5 times less axons in adults than at birth [26], whereas the remaining axons with high electrical activity get thicker myelin sheet [27]. The co-occurring myelination and pruning with other changes in the white matter microstructure during childhood was found to result in a net increase of FA in many brain regions [18]. One of these regions is the corpus callosum, shows early and rapid change of FA with age. Lebel and colleagues [18] found that the genu and splenium of CC reached $90 \%$ of their maximum FA value by 11 years of age. This suggests that in most of our patients, the main part of myelination and pruning in the CC has already finished by the onset of the scoliosis. The found difference between the patient and the control groups, therefore, might indicate that the alteration of white matter microstructure reflected by the FA precedes the spine deformity. Although, both myelination and pruning occur in the adult brain as well $[25,27]$ this has only a small effect on the FA value [18], which further supports that the callosal change is not a consequence of the disease.

A previous morphometry study reported slight difference in the shape of the CC between IS patients and controls [9]. This difference was restricted to a small part of the splenium. In the present study when comparing the FA values of the $\mathrm{CC}$ between groups, we found no difference in the splenium. This suggests that the described shape difference might not affect the microstructure of the CC. This discrepancy might also be explained by the variability in the splenium and its strong age dependence [28] or the different form of scoliosis examined, left thoracic in the study by Wang et al. [9] whereas mainly right thoracic in the present study. Functional MRI was used previously [11] to investigate the brain activity during motor task in IS patients. This study reported different pattern of response in the supplementary motor area (SMA) of patients as compared to controls, and higher asymmetry index. This area is responsible for preparation and execution of movements and participates in the control of posture [29, 30]. The authors, therefore, hypothesized that a defective 
sensorimotor integration at cortical level plays a role in IS pathogenesis. This defect in cortical integration and the more asymmetric activation might be the result of differences in the interhemispheric white matter tracts connecting the motor cortex and SMA. This is in line with our finding, namely that the region of the corpus callosum which shows a significant difference between the two groups is located in the anterior part of the body, which connects mainly the left and right motor and premotor cortices. The altered connections between these cortices can ultimately result in suboptimal interhemispheric balance and control of the corticospinal pathways in IS, since over $30 \%$ of the axons in these tracts arise from the premotor and supplementary motor regions.

\section{Conclusion}

In the current study, we used diffusion tensor imaging to characterize the white matter microstructure of the corpus callosum in patients with idiopathic scoliosis. This is a new way of looking at the abnormalities of the nervous system of this patient group. Although, we had a relatively small number of subjects, we could demonstrate decrease in the FA values of the corpus callosum. These results already indicate the possible involvement of abnormal brain development in the aetiopathogenesis of idiopathic scoliosis; however, further research involving more patients and different types of scoliosis is needed to draw clear conclusions.

To conclude, we have formulated a new testing hypothesis which is the involvement of micro-structural differences in the corpus callosum in idiopathic scoliosis. Our study gives the first hint: a lower FA in idiopathic scoliosis in the body part of the corpus callosum. Future work, using this modality of MR imaging would allow a better understanding of idiopathic scoliosis.

Acknowledgments The authors wish to acknowledge support from Pr JP. Pruvo from the neuro-radiology department (Lille Hospital). This research was supported by La Fondation Yves Cotrel de l'Institut de France (http://fondationcotrel.org). Data from control subjects were obtained from the NIH Pediatric MRI Data Repository created by the NIH MRI Study of Normal Brain Development. This is a multisite, longitudinal study of typically developing children from ages newborn through young adulthood conducted by the Brain Development Cooperative Group and supported by the National Institute of Child Health and Human Development, the National Institute on Drug Abuse, the National Institute of Mental Health, and the National Institute of Neurological Disorders and Stroke (Contract \#s N01HD02-3343, N01-MH9-0002, and N01-NS-9-2314, -2315, -2316, $-2317,-2319$ and -2320 ). A listing of the participating sites and a complete listing of the study investigators can be found at http://pediatricmri.nih.gov/nihpd/info/participating_centers.html. This manuscript reflects the views of the authors and may not reflect the opinions or views of the NIH.

\section{Conflict of interest None.}

Open Access This article is distributed under the terms of the Creative Commons Attribution License which permits any use, distribution, and reproduction in any medium, provided the original author(s) and the source are credited.

\section{References}

1. Parent S, Newton PO, Wenger DR (2005) Adolescent idiopathic scoliosis: etiology, anatomy, natural history, and bracing. Instr Course Lect 54:529-536

2. Miller NH (2007) Genetics of familial idiopathic scoliosis. Clin Orthop Relat Res 462:6-10. doi:10.1097/BLO.0b013e318126c062

3. Gorman KF, Julien C, Moreau A (2012) The genetic epidemiology of idiopathic scoliosis. Eur Spine J. doi:10.1007/s00586012-2389-6

4. Wiener-Vacher SR, Mazda K (1998) Asymmetric otolith vestibulo-ocular responses in children with idiopathic scoliosis. J Pediatr 132:1028-1032

5. Rousie D, Hache JC, Pellerin P et al (1999) Oculomotor, postural, and perceptual asymmetries associated with a common cause. Craniofacial asymmetries and asymmetries in vestibular organ anatomy. Ann NY Acad Sci 871:439-446

6. Beaulieu M, Toulotte C, Gatto L et al (2009) Postural imbalance in non-treated adolescent idiopathic scoliosis at different periods of progression. Eur Spine J 18:38-44. doi:10.1007/s00586-0080831-6

7. Mallau S, Bollini G, Jouve J-L, Assaiante C (2007) Locomotor skills and balance strategies in adolescents idiopathic scoliosis. Spine (Phila Pa 1976) 32:E14-E22. doi:10.1097/01.brs. 0000251069.58498.eb

8. Lambert FM, Malinvaud D, Glaunès J et al (2009) Vestibular asymmetry as the cause of idiopathic scoliosis: a possible answer from Xenopus. J Neurosci 29:12477-12483. doi:10.1523/ JNEUROSCI.2583-09.2009

9. Wang D, Shi L, Chu WCW et al (2009) A comparison of morphometric techniques for studying the shape of the corpus callosum in adolescent idiopathic scoliosis. Neuroimage 45:738-748

10. Wang D, Shi L, Chu WCW et al (2012) Abnormal cerebral cortical thinning pattern in adolescent girls with idiopathic scoliosis. Neuroimage 59:935-942. doi:10.1016/j.neuroimage.2011. 07.097

11. Domenech J, Garcia-Marti G, Marti-Bonmati L et al (2011) Abnormal activation of the motor cortical network in idiopathic scoliosis demonstrated by functional MRI. Eur Spine J 20:1069-1078. doi:10.1007/s00586-011-1776-8

12. Liu T, Chu WCW, Young G et al (2008) MR analysis of regional brain volume in adolescent idiopathic scoliosis: neurological manifestation of a systemic disease. J Magn Reson Imaging 27:732-736. doi:10.1002/jmri.21321

13. Lee JS, Kim S-J, Suh KT et al (2009) Adolescent idiopathic scoliosis may not be associated with brain abnormalities. Acta Radiol 50:941-946

14. Le Bihan D, Breton E, Lallemand D et al (1986) MR imaging of intravoxel incoherent motions: application to diffusion and perfusion in neurologic disorders. Radiology 161:401-407

15. Wakana S, Jiang H, Nagae-Poetscher LM et al (2004) Fiber tractbased atlas of human white matter anatomy. Radiology 230:77-87. doi:10.1148/radiol.2301021640

16. Basser PJ, Pierpaoli C (1998) A simplified method to measure the diffusion tensor from seven MR images. Magn Reson Med 39:928-934 
17. Rousseau M-A, Laporte S, Chavary-Bernier E et al (2007) Reproducibility of measuring the shape and three-dimensional position of cervical vertebrae in upright position using the EOS stereoradiography system. Spine (Phila Pa 1976) 32:2569-2572. doi:10.1097/BRS.0b013e318158cba2

18. Lebel C, Walker L, Leemans A et al (2008) Microstructural maturation of the human brain from childhood to adulthood. Neuroimage 40:1044-1055. doi:10.1016/j.neuroimage.2007.12. 053

19. Wheeler-Kingshott CAM, Cercignani M (2009) About "axial" and "radial" diffusivities. Magn Reson Med 61:1255-1260. doi: $10.1002 / \mathrm{mrm} .21965$

20. Hofer S, Frahm J (2006) Topography of the human corpus callosum revisited-comprehensive fiber tractography using diffusion tensor magnetic resonance imaging. Neuroimage 32:989-994. doi:10.1016/j.neuroimage.2006.05.044

21. Jones DK, Knösche TR, Turner R (2012) White matter integrity, fiber count, and other fallacies: the do's and don'ts of diffusion MRI. Neuroimage. doi:10.1016/j.neuroimage.2012.06.081

22. Metzler-Baddeley C, O’Sullivan MJ, Bells S et al (2012) How and how not to correct for CSF-contamination in diffusion MRI. Neuroimage 59:1394-1403. doi:10.1016/j.neuroimage.2011.08. 043

23. Emery B (2010) Regulation of oligodendrocyte differentiation and myelination. Science (80) 330:779-782. doi:10.1126/science. 1190927
24. Barres BA, Raff MC (1993) Proliferation of oligodendrocyte precursor cells depends on electrical activity in axons. Nature 361:258-260. doi:10.1038/361258a0

25. Yamahachi H, Marik SA, McManus JNJ et al (2009) Rapid axonal sprouting and pruning accompany functional reorganization in primary visual cortex. Neuron 64:719-729. doi:10.1016/j. neuron.2009.11.026

26. LaMantia AS, Rakic P (1990) Axon overproduction and elimination in the corpus callosum of the developing rhesus monkey. J Neurosci 10:2156-2175

27. Ishibashi T, Dakin KA, Stevens B et al (2006) Astrocytes promote myelination in response to electrical impulses. Neuron 49:823-832. doi:10.1016/j.neuron.2006.02.006

28. Luders E, Thompson PM, Toga AW (2010) The development of the corpus callosum in the healthy human brain. J Neurosci 30:10985-10990. doi:10.1523/JNEUROSCI.5122-09.2010

29. Tanji J (1994) The supplementary motor area in the cerebral cortex. Neurosci Res 19:251-268

30. Deiber MP, Honda M, Ibanez V et al (1999) Mesial motor areas in self-initiated versus externally triggered movements examined with fMRI: effect of movement type and rate. J Neurophysiol 81:3065-3077 